\title{
Analysis of Network Robustness in Weighted and Unweighted Approaches: A Case Study of the Air Transport Network in the Belt and Road Region
}

\author{
Lu Zhang $\mathbb{D}^{1},{ }^{1}$ Yannan Zhao $\left(\mathbb{D},{ }^{2}\right.$ Dongli Chen $\left(\mathbb{D},{ }^{3}\right.$ and Xinhuan Zhang ${ }^{4}$ \\ ${ }^{1}$ Beijing Technology and Business University, School of International Economics and Management, Beijing 100048, China \\ ${ }^{2}$ Institute of Geographic Sciences and Natural Resources Research, Chinese Academy of Sciences, \\ Chaoyang District, Beijing 100101, China \\ ${ }^{3}$ School of Cultural Industry and Tourism Management, Henan Univerisity, Kaifeng 475001, China \\ ${ }^{4}$ State Key Laboratory of Desert and Oasis Ecology, Xinjiang Institute of Ecology and Geography, \\ Chinese Academy of Sciences, Urumqi 830011, China
}

Correspondence should be addressed to Yannan Zhao; zhaoyn.16b@igsnrr.ac.cn and Dongli Chen; 1181532817@qq.com

Received 21 June 2020; Revised 4 April 2021; Accepted 23 May 2021; Published 3 June 2021

Academic Editor: Michela Le Pira

Copyright $\odot 2021 \mathrm{Lu}$ Zhang et al. This is an open access article distributed under the Creative Commons Attribution License, which permits unrestricted use, distribution, and reproduction in any medium, provided the original work is properly cited.

Aviation transport is one of the most important and critical infrastructures in today's global economy. Failure in its proper operations can seriously impact regional economic development, which is why it is important to evaluate network robustness. Previous analyses of robustness have mainly been conducted with an unweighted approach. In the development of air transport, however, the demand for route configuration has gradually decreased, while the demand for flight adjustments has increased. Consequently, the aviation network has developed unevenly, so adhering to a uniform approach for evaluating network robustness may lead to inaccurate results. Therefore, we examined which centrality sequence is the most sensitive to network robustness in both unweighted and weighted approaches. The air transport network selected for the case study comprised the six subregions of the Eurasian landmass of the Belt and Road region. The study results showed the following: (a) in the network constructed as an unweighted one, betweenness, and degree centrality had higher priorities in preserving network functionalities than eigenvector and closeness centrality; (b) in the network constructed as a weighted one, recursive power had a higher priority in preserving network functionalities than recursive centrality; and (c) no particular centrality measurement had a significant advantage in representing the totality of robustness. The betweenness centrality sequence was sensitive to the average shortest path length and global efficiency; the recursive power sequence was sensitive to the clustering coefficient, while degree centrality was sensitive to graph diversity. The findings of this study support the decisions about managing air transportation in the Belt and Road region.

\section{Introduction}

Geographically, air transportation infrastructure has linked far-flung locations and fostered intercity relations [1]. The development of air transportation services in the past few decades has played a vital role in economic growth and prosperity by making countries or regions accessible to large markets and by increasing the influx of investments [2-4]. However, failures or inefficiencies in the system, potentially caused by extreme bad weather, natural disasters, terrorist attacks, and so on, could lead to disruptions in flight operations and create large losses to the general economy [5-7]. For instance, bad weather has been identified as the most common external disruptive agent [8]. Moreover, the emergency shutdown of air movement caused by the COVID19 pandemic has greatly reduced gross operating revenues for airlines worldwide [9]. Modern states and societies rely on the availability, reliability, and safety of air transport infrastructure, and thus air transport infrastructure has been a major concern for policymakers and scholars.

The ability of a transportation system to maintain its designed functionality in the cases of disturbances is defined 
as robustness [10]. The rise of complex networks, where nodes refer to airports and edges to the links connecting them, offered new insights into the analysis of networks in a variety of real-world systems [11]. Thus, nodes and edges have become the main focus of integrated management of air transport networks $[12,13]$. Numerical simulations and analytical approaches have been proposed for detecting key nodes during random or targeted attacks [14-16]. For instance, node centralities (e.g., degree, betweenness, and closeness centrality) have been widely used to reflect the importance of nodes [17-19]. Nodes with high centrality are considered important in preserving network functionalities. However, it is noteworthy that these centrality measurements focus on the unweighted approach by assuming the strengths of the connections are uniform. With the development of air transport, the demand for route configuration gradually has decreased, while the demand for flight adjustments has increased. As a result, links between two large airports can differ greatly from those between two small airports. This has a significant impact on the network's robustness. Thus, a uniform approach to evaluating network robustness may lead to inaccurate results.

Recent measurements in a weighted approach have been conducted by considering link weight [20,21]. Other studies identified the key nodes through a weighted approach by introducing Bonacich power centrality [16], heterogeneous degrees [22], and recursive centrality and power [23]. However, what has rarely been noticed is the gap in the ability of weighted and unweighted approaches to preserve network functionalities. Correctly determining this gap will help understand the functionality of air transportation, especially in the cases where the link weight is not available. Therefore, the first aim of this paper was to explore the network robustness through unweighted and weighted approaches.

One weighted network is the air transport network in the Belt and Road (B\&R), where flights and routes are distributed unevenly [23]. Interest in the B\&R initiative has grown since 2013 when it was first proposed. On the one hand, the B\&R can be seen as a configuration to reduce spatial barriers, particularly by developing transport infrastructure to facilitate flows across city nodes [24, 25]. On the other hand, the demands for air transportation services come from the increasing requirements of high-quality, speedy, and reliable international transport. Air transportation can enable efficient processing and transmission of information and face-to-face contacts, which are extremely important for transnational businesses [1, 26-28]. As a result, there has been a rapid and uneven rise in air transport in the B\&R region in the past few years. For instance, East Asia has the highest number of flights per week (1308). This is 10.8 times the number of weekly flights in Central Asia (121). As air transport is becoming increasingly critical, studies on the air transport network in the $\mathrm{B} \& \mathrm{R}$ (ATNBR) has been receiving an increasing amount of interest $[29,30]$.

However, for various reasons, including geopolitics and natural disasters, the ATNBR is often subject to various attacks, resulting in significant losses. For example, due to its geopolitical importance, Tel Aviv airport has been the focus of terrorist attacks, which have caused great losses to local and global gross domestic product (GDP). Thus, the analysis of the robustness of the air transport network has significance due to its contribution towards increasing profits and economic growth in the B\&R. Until now, the study of the robustness of ATNBR has been a relatively unexplored field. Under the context of the $\mathrm{B} \& \mathrm{R}$ initiative, the following question has arisen: Which centrality sequence has the most impact on preserving network functionalities in this region? Thus, the second aim of this paper was to explore the air transport network robustness in the $\mathrm{B} \& \mathrm{R}$ region, which will facilitate beneficial references for evidence-based configurations.

Focusing on the two-fold aim of this study, the rest of the paper is divided into four sections. Section 2 introduces the methodology applied for this analysis. Section 3 reports the main findings of our analysis. Section 4 discusses the results, after which the study is concluded in Section 5 with the presentation of a summary of the study results.

\section{Methodology}

2.1. Study Area. Although the B\&R region is an exoteric, international economic cooperation network without any precise scope [31], the research scope is always confined to 66 countries on the list issued by the Chinese government in 2016 (Table 1). In 2017, the population and economy of the $\mathrm{B} \& \mathrm{R}$ countries amounted to $55 \%$ and $31.7 \%$ of the global value, respectively. Besides, the critical role of air transport in this region is becoming increasingly prominent due to two aspects. First, air transport has a lower entry threshold compared to railway and highway transportation. The B\&R region covers a wide area of the Eurasian landmass where the topography is quite complicated. Moreover, air transport is less environmentally sensitive, and the shielding effect of breaking through the natural boundary is significant. Second, air transport is more accessible than other traffic modes. The $\mathrm{B} \& \mathrm{R}$ region spans more than 10,000 kilometers from south to north and east to west. The broad area of the $\mathrm{B} \& \mathrm{R}$ region has increased the demands on traffic due to the reason of speed, especially in poor regions where high-speed transportation is limited [32]. Thus, air transportation plays a critical role in implementing the B\&R initiative because of its unique advantages in breaking through the shielding effect generated by natural boundaries, its speed, and its ability to meet the requirements for long-distance access.

Data on weekly intercity flights can be used to map the intercity transport linkages. The dataset was gathered in the first week of August 2018 using Google Flight, and each record contained information on flights, including schedule, and the airline. Ideally, directed and annual data should be used; however, due to time constraints, our dataset was much smaller. Our overall goal was to estimate the weight differences in edges. From the information collected on several weekly flights in August, we found the following: (a) all binary connections were retrieved and (b) demand did not show either a peak or a trough. Thus, we believe this effect to be of minor relevance. 
TABLE 1: Country list in B\&R region

Subregion
East Asia
Central Asia
Southeast Asia
South Asia
Central and Eastern Europe (Even if the sub-regional division of
Europe is not confirmed to the Europe view, which considers Greece
as a part of Southern Europe or middle part of Europe, the official
publications of the Chinese government integrate Greece in the list
of Central and Eastern Europe)

West Asia

Turkey, Bahrain, Kuwait, Oman, Qatar, Saudi Arabia, The United

Arab Emirates, Yemen, Armenia, Azerbaijan, Georgia, Iraq, Iran, Israel, Jordan, Lebanon, Syria, Egypt, Cyprus

The criteria used for including a B\&R city in the study are as follows: (a) it was in the study area, covering six subregions of the Eurasian landmass (East, West, South, Southeast, and Central Asia, and Central and Eastern Europe). This includes 66 countries (Table 1) where there were 1718 airports; (b) there was at least one airport that hosted 25 or more airlines; (c) it was a capital of one of the 66 countries, even if it did not host 25 or more airlines. In the end, 198 B\&R cities were selected. Among them, 147,970 nonstop flights linking 2717 distinct pairs of nodes took place in one week (Figure 1). Thus, the ATNBR consists of 198 cities (nodes), 2717 edges in its unweighted network, and 147,970 connections in its weighted network.

2.2. Robustness Assessment Approach. To compare network robustness in weighted and unweighted approaches, centrality measures were introduced. Several centrality measures have been advocated in the analysis of unweighted networks, out of which degree, betweenness, eigenvector, and closeness centralities have been the most widely used ones. Recursive power and centrality were introduced to identify nodes that were the most critical and central in the weighted networks.

2.2.1. Degree Centrality. Degree centrality refers to the number of edges to which a given node is connected. Thus, a node with a high degree of centrality is directly connected to many nodes. In an adjacency matrix, if there were edges between nodew $i$ and $j$, then $A_{i j}=1$; otherwise, $A_{i j}=0$. Thus, the degree centrality $D_{i}$ of node $i$ is given by the following formula:

$$
D_{i}=\sum_{j=1}^{n} A_{i j}
$$

2.2.2. Eigenvector Centrality. Eigenvector centrality is another widely applied centrality measure. It refers to the refinement of degree centrality. The difference between the two centrality measures is that a node is considered to have a high degree of centrality if it is connected to many other nodes, while a node is considered to have high eigenvector centrality if it is linked to other nodes that themselves have high-degree centrality [33]. Referring to the measures presented by [34], the eigenvector centrality of node $i$ is given as follows:

$$
e_{i}=\frac{1}{\rho} \sum_{j=1}^{n} A_{i j} e_{j}
$$

where $\rho$ is a constant, $A_{i j}$ is the adjacency value of nodes $i$ and $j$, and $e_{i}$ and $e_{j}$ refer to the eigenvector centrality of nodes $i$ and $j$, respectively. It is assumed that the eigenvector centrality of each node is nonnegative, indicating that $e_{i} \geq 0$.

In matrix notation with $e=\left(e_{1}, \ldots, e_{n}\right)$, this yields

$$
\rho e=A e .
$$

This type of equation is well known and solved by the eigenvalues and eigenvectors of $A$.

2.2.3. Closeness Centrality. In general, closeness centrality refers to the mean geodesic path between nodes in networks. Here, the geodesic path is defined as the number of edges traversed from node $i$ to $j$. Thus, a node with a high closeness centrality indicates a short communication edge to other nodes in networks. In general, geodesic paths are not unique, as there can be several paths between two given nodes with the same shortest length. However, at least one geodesic path always exists between any two nodes in the same connected component of a network. The mean geodesic distance between $i$ and all other nodes in the network is given by

$$
g_{i}=\frac{1}{N} \sum_{j=1}^{n} \gamma_{i j}
$$

Here, $\gamma_{i j}$ refers to the number of edges traversed from node $i$ to $j$. Then, the closeness centrality $c_{i}$ of node $i$ is defined by Iyer et al. [33] as follows:

$$
c_{i}=\frac{1}{g_{i}}=\frac{N}{\sum_{j=1}^{n} \gamma_{i j}} .
$$

2.2.4. Betweenness Centrality. Another notion of centrality is betweenness centrality, which measures the number of short paths between nodes while they pass through a given node. Thus, if we define 


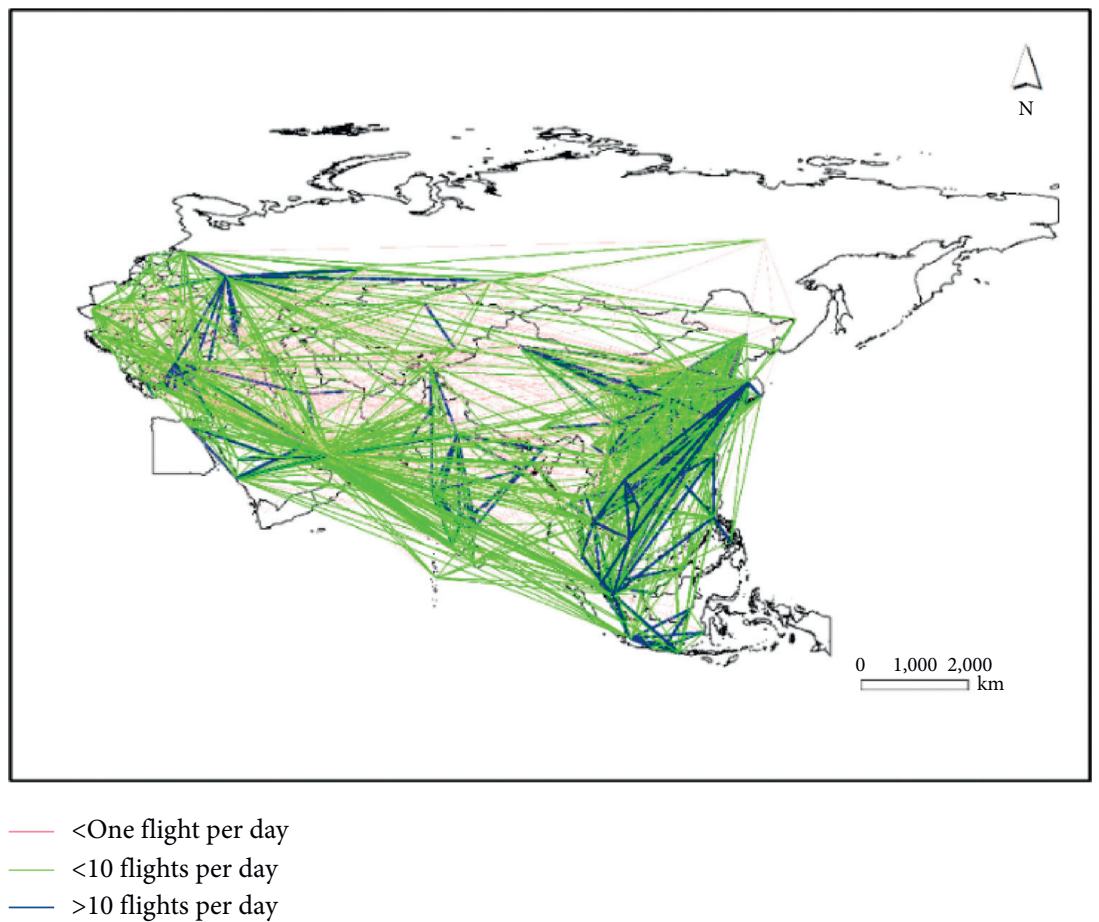

Figure 1: Flight distribution in the study area [30].

$v_{i}(s, t)=\left(\begin{array}{cc}1, & \text { if } i \text { lies on the geodesic path from } s \text { to } t \\ 0, & \text { otherwise. }\end{array}\right)$

Then the betweenness centrality $b_{i}$ of node $i$ is given by Iyer et al. [33] as follows:

$$
b_{i}=\sum_{s, t \in N} v_{i}(s, t)
$$

2.2.5. Recursive Centrality. In weighted networks, measures to differentiate power and centrality were developed by Neal [35]. Accordingly, the recursive centrality $\left(R C_{i}\right)$ of node $i$ can be computed as follows:

$$
R C_{i}=\sum_{j=1}^{n} R_{i j} * D_{j}
$$

where $D_{j}$ refers to the degree of centrality of node $j$ and $R_{i j}$ refers to the strength of the connection based on weekly flights between nodes $i$ and $j$. Accordingly, recursive centrality refers to the strength of node $i$, which is calculated by the degree of centrality of the node to which it is connected. The recursive power of node $i$ weighs each connection by the contact's inverse degree of centrality.

2.2.6. Recursive Power. Similar to calculating recursive centrality, recursive power $\left(\mathrm{RP}_{i}\right)$ of node $i$ can be computed as follows [35]:

$$
\mathrm{RP}_{i}=\sum_{j=1}^{n} \frac{R_{i j}}{D_{j}}
$$

Thus, recursive power reflects the extent to which node $i$ is dominating node $j$. In general, a node with high recursive power exercises a great influence over the facilitation of opportunities since it always connects to cities that are poorly connected. Consequently, when powerful nodes fail, they cause the nodes that connect to the network to fail too.

2.3. Attack Strategies. The critical nodes will be detected through node isolation through adaptive strategies (see Figure 2). The first node to be removed is the node with the highest value calculated using one of the centrality measures. Once the node and its links are deleted, the new adjacency matrix gets constructed. If a link exists in the new network, then the next node to be removed is the node with the first value calculated under the same centrality measures. Then, the operation of the entire network enters a loop. The loop continues until no link exists in the new networks.

The response of the ATNBR under targeted and random attacks was explored to show the robustness of the network. Our analysis considered seven strategies for progressive node removal, namely, (a) degree-based, (b) closenessbased, (c) betweenness-based, (d) eigenvector-based, (e) recursive power-based, (f) recursive centrality-based, and (g) random node removal. In each case, we first removed the node with the highest centrality value. Then, we continued selecting and removing nodes in the descending order of their centrality value. 


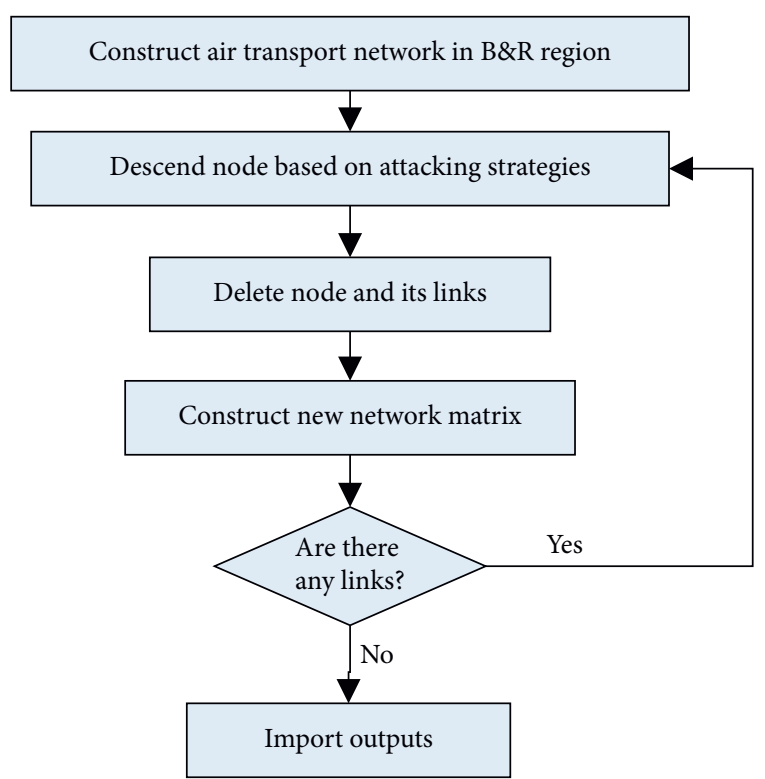

FIGURE 2: Flowchart of attacking strategy.

2.4. Robustness Metrics. In previous studies, numerical metrics were proposed for calculating network robustness [36]. These metrics were classified into two groups: those based on node centrality and on network structure, both of which measure the strength of the entire network. (a) Node centrality included node degree, closeness, betweenness centrality, and clustering coefficient, which refers to the quantity and quality of one node's connections to its neighbors, and its importance in the entire network. Note that, in this type, only the clustering coefficient is used as a metric of robustness. (b) The network structure metric included the size of the giant component, average shortest path length, and global efficiency. The size of the giant component does not account for the distances between node pairs. For this reason and considering the obvious drawback of the size of the giant component, only the other two metrics were selected. Finally, graph diversity was chosen since it presents the ability of a graph to remain connected. Therefore, four metrics, namely, clustering coefficient (C), average shortest path length $(\mathrm{L})$, graph diversity $(\mathrm{G})$, and global efficiency $(\mathrm{E})$, were selected in this study.

2.4.1. Clustering Coefficient. The clustering coefficient is used to assess how the neighbors of a node are connected. It is defined as the clustering degree in a graph [37]. Node $i$ 's clustering coefficient represents the probability that two nodes are connected to a particular one [38]. It is computed as follows:

$$
C_{i}=\frac{E_{i}}{k_{i}\left(k_{i}-1\right) / 2}
$$

where $E_{i}$ represents the number of edges that are directly connected to node $i$ and $k_{i}$ illustrates the degree of node $i$. As a result, the clustering coefficient includes the average values of the clustering coefficient of the nodes. According to Liu et al. [17], the clustering coefficient is given by the following:

$$
C=\frac{1}{N} \sum_{i=1}^{n} C_{i}
$$

2.4.2. Average Shortest Path Length. The average path length is the average number of edges along the shortest path between all pairs of nodes [37]. As we were aiming to construct the undirected network in this analysis, the average path length $(L)$ is calculated by the following:

$$
L=\frac{1}{N(N-1) / 2} \sum_{i>j}^{N} d_{i j},
$$

where $d_{i j}$ refers to the number of edges along the shortest path between nodes $i$ and $j$ and $N$ is the total number of nodes in the network. A low value of $L$ indicates that the nodes in a network are more accessible with fewer transfers, which means the network has higher robustness.

2.4.3. Graph Diversity. Since it considers all the possible paths between two nodes, graph diversity is related to the number of nodes shared by the shortest path. Total graph diversity is the average of the diversity on all valid paths [39]. A larger total graph diversity indicates stronger robustness.

Let the shortest path between a pair of vertices $(i, j)$ be $P_{0}$. Then, for any other path $P_{k}$ between the same source and destination, we defined the diversity function $D\left(P_{k}\right)$ with respect to $P_{0}$ as follows:

$$
D\left(P_{k}\right)=1-\frac{\left|P_{k} \cap P_{0}\right|}{\left|P_{0}\right|} .
$$

The path diversity gives a value of 1 , if $P_{k}$ and $P_{0}$ are completely disjoint, and a value of 0 , if $P_{k}$ and $P_{0}$ are identical. Then, the effective path diversity (EPD) is calculated as follows:

$$
\mathrm{EDP}=1-e^{-\beth k_{i j}}
$$

where $k_{i j}$ is a measure of the added diversity, which is defined as follows:

$$
k_{i j}=\sum_{i=1}^{k} D_{\min }\left(P_{i}\right),
$$

where $D_{\min }\left(P_{i}\right)$ is the minimum diversity of path $i$ when evaluated against each member of the set of paths $\left\{P_{1} \ldots P_{i-1}\right\}$ previously selected for that pair of nodes. $\lambda$ is an experimentally determined constant that scales the impact of $k_{i j}$ based on the utility of this added diversity. The total graph diversity can now be defined as the average of the EPD values of all node pairs within that graph.

2.4.4. Global Efficiency. The concept of global efficiency is widely used to optimize transportation systems and connectivity. From Latora and Marchiori [40], it can be said that global efficiency is calculated as follows: 


$$
E=\frac{1}{N(N-1)} \sum_{i \neq j \in G} \frac{1}{d_{i j}},
$$

where $d_{i j}$ refers to the shortest path between node $i$ and node $j$.

\section{Results}

The primary aim of this analysis was to compare the differences in robustness under various centrality sequences and to explore which centrality sequence had the most effect on preserving network functionalities in the $\mathrm{B} \& \mathrm{R}$ region. Based on this twofold aim, the sequential removal of the nodes was based on the centrality ranking of the nodes. Then, our analysis computed the change of some major metrics in preserving network functionalities by removing nodes in the decreasing order of its specified centrality measurements.

3.1. Correlation Analysis. Before focusing on the main aims of this analysis, the correlation analysis of centrality measures in unweighted and weighted networks was conducted to gain some insights into the relationships between these centrality measures, as described later in Section 4. In Figures 3 and 4, each data point in the plots represented the value of the different centralities for the original network, before the performance of any form of attack strategy. After conducting several tests, linear correlation regression was observed to have the best performance of $R^{2}$, which aims to evaluate regression accuracy. In general, the high value of $R^{2}$ represents a strong correlation among these parameters. Note that two outcomes can be observed.

First, the regression between recursive power in the weighted network and four centrality measures in the unweighted networks were evaluated (see Figure 3). In brief, the $R^{2}$ values for evaluating the regression accuracy between recursive power and degree centrality, betweenness centrality, and closeness centrality were found to be $0.73,0.77$, and 0.67 , respectively. This meant that a node with a high value of recursive power tended to have high values in degree, betweenness, and closeness centrality as well. Among these, recursive power had the strongest linear correlation with betweenness centrality (Figure 3(b)). By comparison, Figure $3(\mathrm{~d})$ shows a node with high recursive power and low eigenvector centrality, indicating that the correlation between them is relatively low (0.35).

Second, the results of the regression between recursive centrality and four centrality measures were examined (see Figure 4). The results showed that recursive centrality had the strongest linear regression relation with eigenvector centrality $\left(R^{2}=0.97\right)$ (Figure $\left.4(\mathrm{~d})\right)$. The recursive centrality also had strong linear regression correlation with degree centrality $\left(R^{2}=0.83\right)$ (Figure $\left.4(\mathrm{a})\right)$ and closeness centrality $\left(R^{2}=0.56\right)$ (Figure $4(\mathrm{c})$ ). By comparison, the $R^{2}$ value for the regression accuracy between recursive centrality and betweenness centrality was only 0.26 (Figure 4(b)), indicating that a weak linear correlation existed.
3.2. Sequential-Targeted Attacking. The measurements of robustness metrics in sequential-targeted attacking have been presented, and a preliminary robustness comparison was conducted. The results are presented in this section. For the first $20 \%$ nodes in each centrality measure, see Table 2.

In general, the clustering coefficient decreased with the removal of the nodes. Thus, the node sequence that decreased most sharply had the least vulnerable network robustness. Figure 5 shows the effects of the attack on $20 \%$ of the first nodes on clustering coefficients. In brief, the ATNBR was highly resistant to random attacks but very vulnerable to targeted attacks on nodes. In particular, the four centrality measures in unweighted networks and the two centrality measures in weighted networks displayed great volatility. For instance, in unweighted networks, nodes following the ranking for degree centrality were more robust and had a higher tolerance to attack than any other sequences at first. However, with the continuous isolation of nodes, eigenvector centrality became the best indicator of robustness. In contrast, nodes following the ranking for betweenness centrality showed the least robustness. In weighted networks, the results showed that the network in terms of recursive power was less robust than that of recursive centrality. In the comparison of all centrality measures, recursive power was observed to be the least robust sequence. Nodes following degree centrality, eigenvector centrality, and recursive centrality showed the highest tolerance for targeted attacks. Moreover, while comparing all the centrality measures in the two networks, we found that the nodes following recursive power and betweenness centrality ranking were less robust than when following other rankings, and they respond well to the correlation results in Section 3.1.

The average shortest path length increased with the removal of each node. The node sequence that increased this length most sharply had the least vulnerable network robustness. Figure 6 shows the effects on the average shortest path length. In brief, the average shortest path increased as much as $20 \%$ of isolation nodes. The network was found to be highly resistant to random attacks but very vulnerable to targeted attacks on nodes. In unweighted networks, the nodes following the betweenness centrality ranking were found to be the least robust and had the lowest tolerance for attacks on all the sequences. The average shortest path length increased the most slowly in the sequence of eigenvector centrality, indicating that that sequence had the highest network robustness. Among all the centrality measures, the network modified in terms of betweenness centrality showed the least robustness. While comparing all the centrality measures in the two networks, the nodes following recursive power and betweenness centrality ranking were observed to be the two least robust groups than those following other rankings, aligning well with the correlation results in Section 3.1.

Graph diversity decreases as nodes are removed, indicating that the node sequence that decreased the most sharply had the least vulnerable network robustness. Figure 7 shows the robustness results for graph diversity under sequential targeted attacking. In brief, networks were degraded differently under a sequential-targeted attack. The following three 


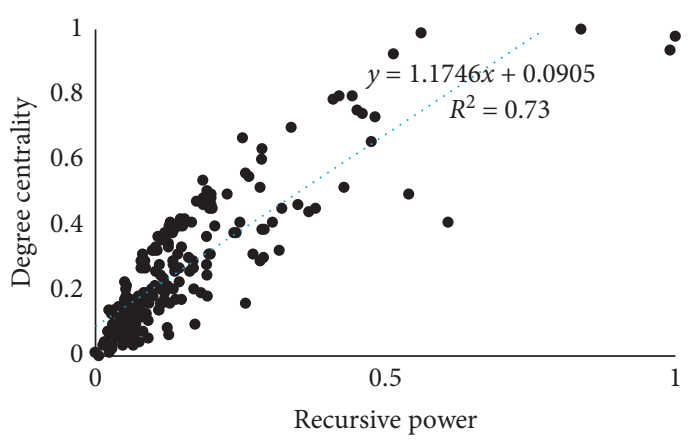

(a)

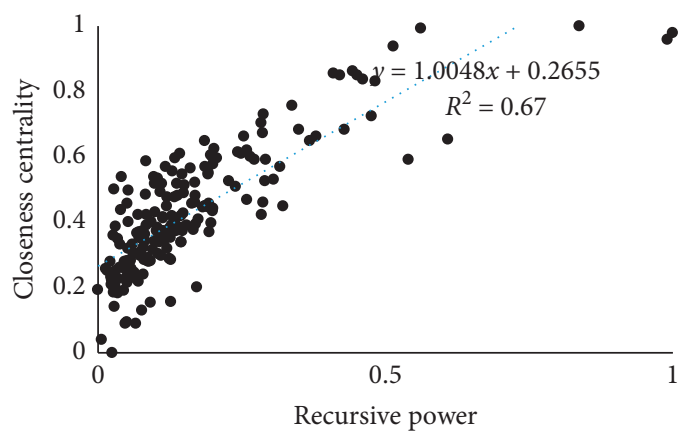

(c)

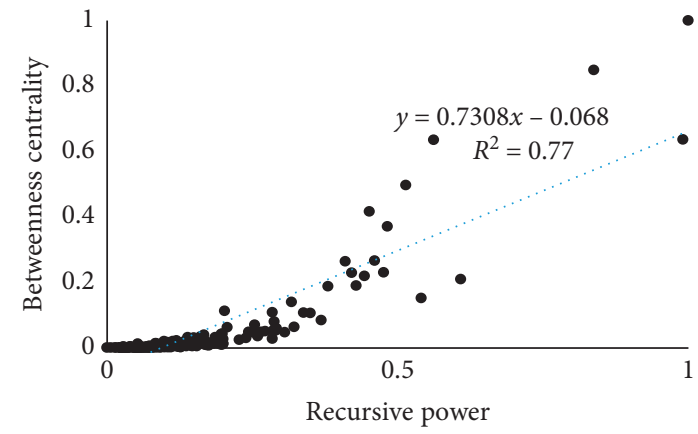

(b)

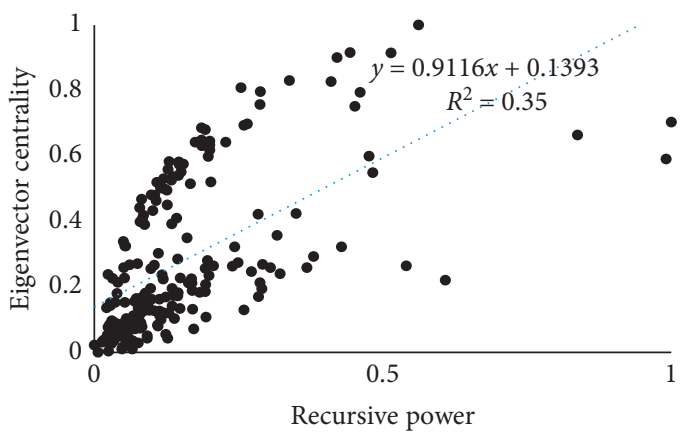

(d)

FIGURE 3: Linear correlation regression between recursive power and four centrality measures: (a) degree centrality, (b) betweenness centrality, (c) closeness centrality, and (d) eigenvector centrality.

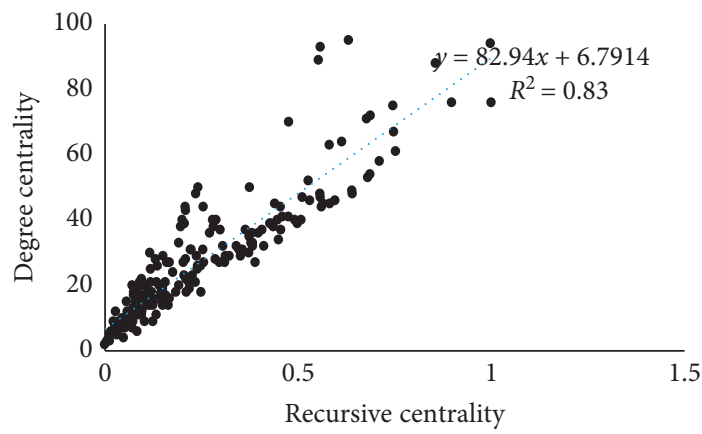

(a)

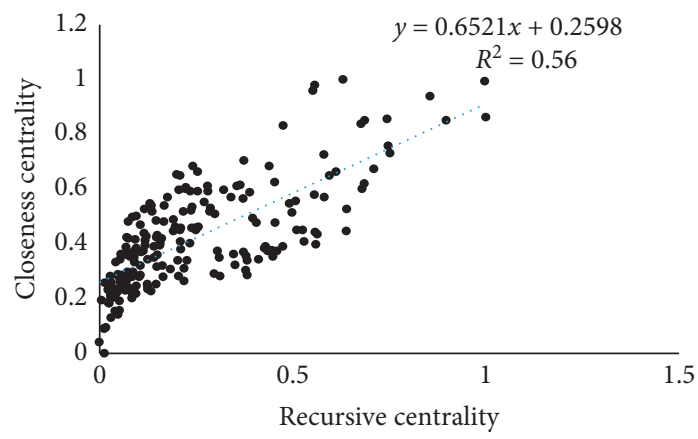

(c)

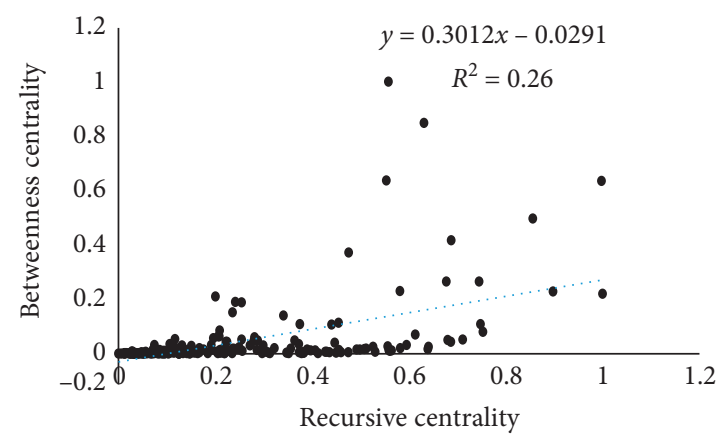

(b)

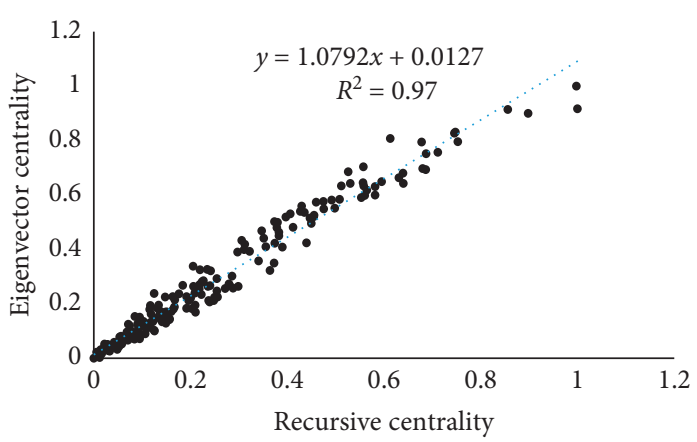

(d)

Figure 4: Correlation between recursive centrality and four other measures of centrality: (a) degree centrality, (b) betweenness centrality, (c) closeness centrality, and (d) eigenvector centrality. 
TABle 2: Cities listed by centrality in B\&R region.

\begin{tabular}{|c|c|c|c|c|c|c|c|}
\hline No. & Degree & Betweenness & Recursive power & No. & Degree & Betweenness & Recursive power \\
\hline 1 & Dubai & Moscow & Moscow & 21 & Abu Dhabi & Delhi & Jakarta \\
\hline 2 & Beijing & Dubai & Istanbul & 22 & Xiamen & Saint Petersburg & Kuwait \\
\hline 3 & Moscow & Istanbul & Dubai & 23 & Athens & Chengdu & Jeddah \\
\hline 4 & Istanbul & Beijing & Warsaw & 24 & Tianjin & Taipei & Novosibirsk \\
\hline 5 & Bangkok & Bangkok & Beijing & 25 & Hangzhou & Sharjah & Chengdu \\
\hline 6 & Guangzhou & Singapore & Athens & 26 & Shenyang & Baku & Nicosia \\
\hline 7 & Shanghai & Doha & Bangkok & 27 & Changsha & Jeddah & Chongqing \\
\hline 8 & Hong Kong & Seoul & Doha & 28 & Wuhan & Nicosia & Belgrade \\
\hline 9 & Singapore & Hong Kong & Kuala Lumpur & 29 & Guiyang & Novosibirsk & Abu Dhabi \\
\hline 10 & Seoul & Kuala Lumpur & Seoul & 30 & Delhi & Chongqing & Cairo \\
\hline 11 & Doha & Guangzhou & Singapore & 31 & Qingdao & Cairo & Xi'an \\
\hline 12 & Kunming & Shanghai & Shanghai & 32 & Zhengzhou & Xi'an & Shenzhen \\
\hline 13 & Taipei & Warsaw & Prague & 33 & Jerusalem & Mumbai & Zagreb \\
\hline 14 & Kuala Lumpur & Prague & Guangzhou & 34 & Urumqi & Kuwait & Taipei \\
\hline 15 & Chengdu & Jerusalem & Hong Kong & 35 & Sharjah & Riyadh & Riyadh \\
\hline 16 & Chongqing & Athens & Jerusalem & 36 & Nanjing & Tel Aviv Yafo & Mumbai \\
\hline 17 & Shenzhen & Jakarta & Saint Petersburg & 37 & Saint Petersburg & Shenzhen & Muscat \\
\hline 18 & Xi’an & Urumqi & Delhi & 38 & Taiyuan & Tehran & Hangzhou \\
\hline 19 & Nanning & Abu Dhabi & Kunming & 39 & Nanchang & Harbin & Baku \\
\hline 20 & Prague & Kunming & Sharjah & 40 & Warsaw & Zagreb & Urumqi \\
\hline
\end{tabular}

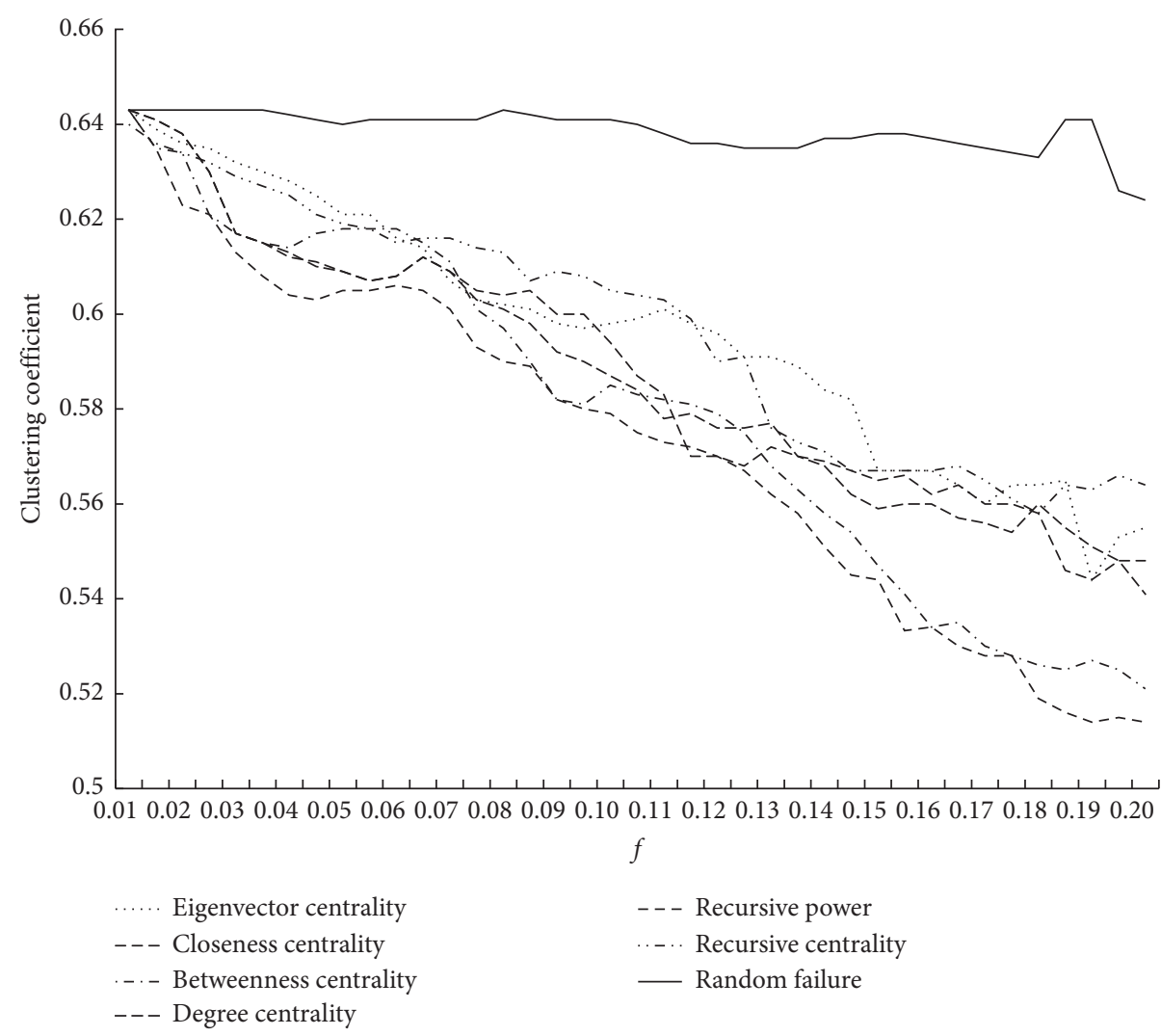

FIGURE 5: Evolution of the clustering coefficients in unweighted and weighted networks. $f$ refers to the percentage of nodes removed.

observations can be made. First, networks exhibit greater vulnerability to sequential attacks based on any centrality measure than under a random attack. Second, in unweighted networks, there were only slight differences in the effectiveness of sequential targeted attacking the first 20\% nodes. However, the nodes following the degree centrality ranking were the least robust and had the lowest tolerance to attacks than the other sequences. In contrast, node sequence in degree centrality ranking showed the highest robustness at first. However, as nodes were isolated continually, eigenvector centrality was observed to be the most robust sequence. Third, in weighted networks, the network in terms of recursive power was less robust than that of recursive centrality. In the comparison of all the centrality measures in unweighted and 


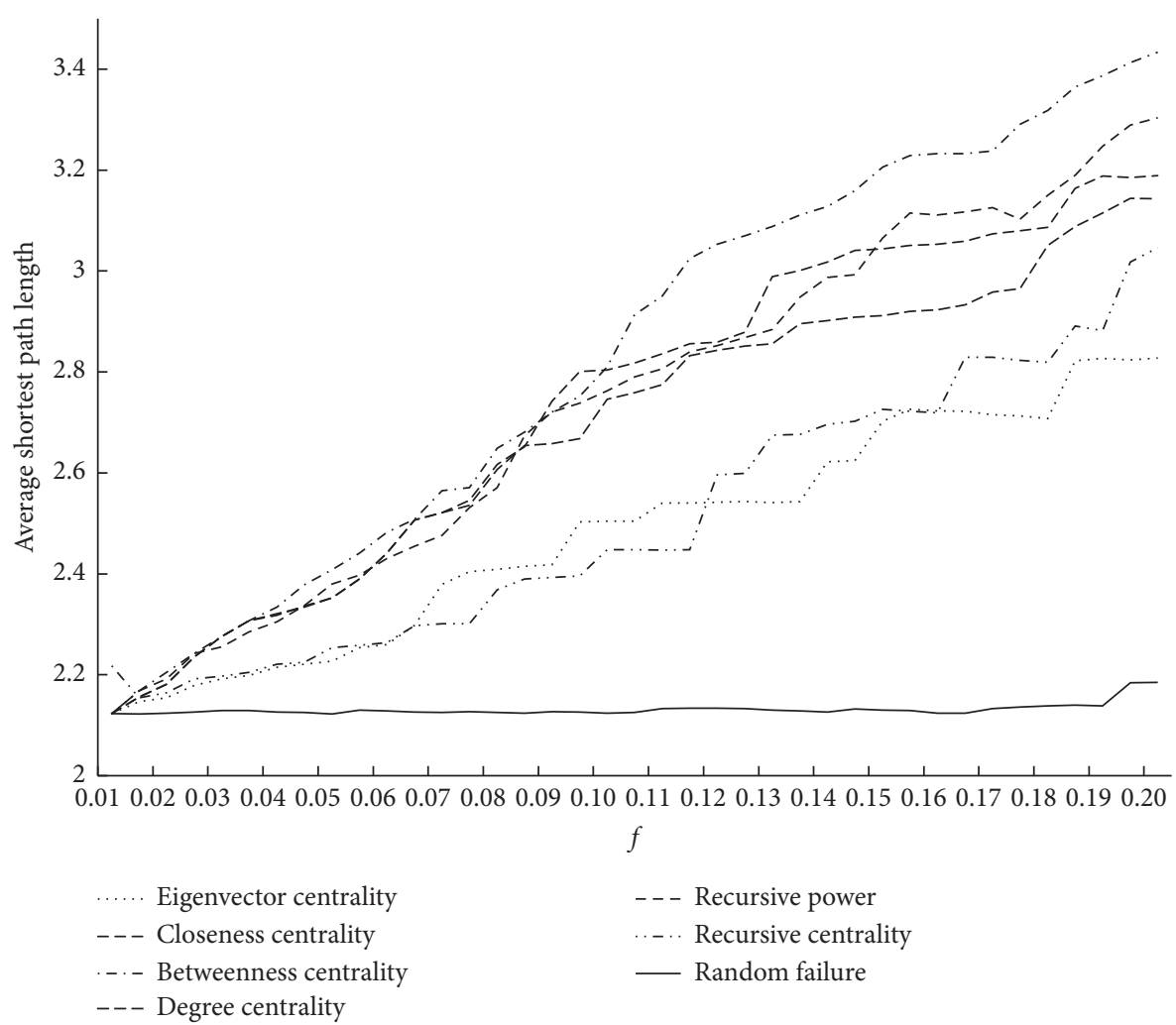

FIGURE 6: Evolution of the average shortest path length in unweighted and weighted networks.

weighted networks, nodes following the degree centrality ranking showed the least robustness.

Global efficiency decreases as the nodes are removed, indicating that the node sequence that decreased the most sharply had the least vulnerable network robustness (see Figure 8). There were two initial observations. First, in unweighted networks, the nodes ranking in the betweenness centrality sequence were the least robust of the sequences. In contrast, nodes ranking in the eigenvector centrality sequence showed the most robustness in facing targeted attacks. Second, in weighted networks, the network in terms of recursive power was less robust than that of recursive centrality. In the comparison of all the centrality measures in unweighted and weighted networks, it can be found that the betweenness centrality ranking was the most robust of all the sequences. Besides, it is noteworthy that global efficiency isolated by random sequence did not provide the highest resistance to attacks, as already shown in other robustness metrics. Node sequences following degree centrality ranking showed the highest robustness. While comparing all the centrality measures in the two networks, the nodes following recursive power and betweenness centrality ranking were observed to be less robust than those following other rankings, responding well to the correlation results presented in Section 3.1.

\section{Discussion}

The failure of a node has a significant impact on robustness, depending on the element affected. Thus, detecting key elements of network robustness is of great importance. Ample studies have examined the robustness of both model and real networks experiencing either errors or attacks $[41,42]$. This analysis explored how the structure of a complex network removes nodes with simultaneous and sequential-targeted attacks based on degrees, betweenness, and closeness centralities in unweighted networks. However, for the majority of networks, the most vulnerable nodes are often related to their strengths in a weighted network, given the imbalance in their internal strength distribution. Therefore, our results also extend the previously discovered targets based only on the centrality and nature of the unweighted network.

Table 3 summarizes the centrality measures with the least robustness metrics. Specifically, nodes ranked by the betweenness centrality had the least robustness in terms of the average shortest path length and global efficiency. Nodes in the recursive power sequence are associated with the least robustness from the aspect of the clustering coefficient. Moreover, nodes ranking in the degree centrality sequence provided the least robustness in terms of graph diversity. This analysis showed that the critical nodes in ATNBR can be detected through adaptive strategies based on the betweenness centrality, degree centrality, and recursive power. Thus, different centrality measures reflect different aspects of network robustness, such that no one centrality measurement has significant advantages over the others in representing the totality of robustness.

In general, nodes following either recursive power or betweenness centrality ranking were found to be less robust 


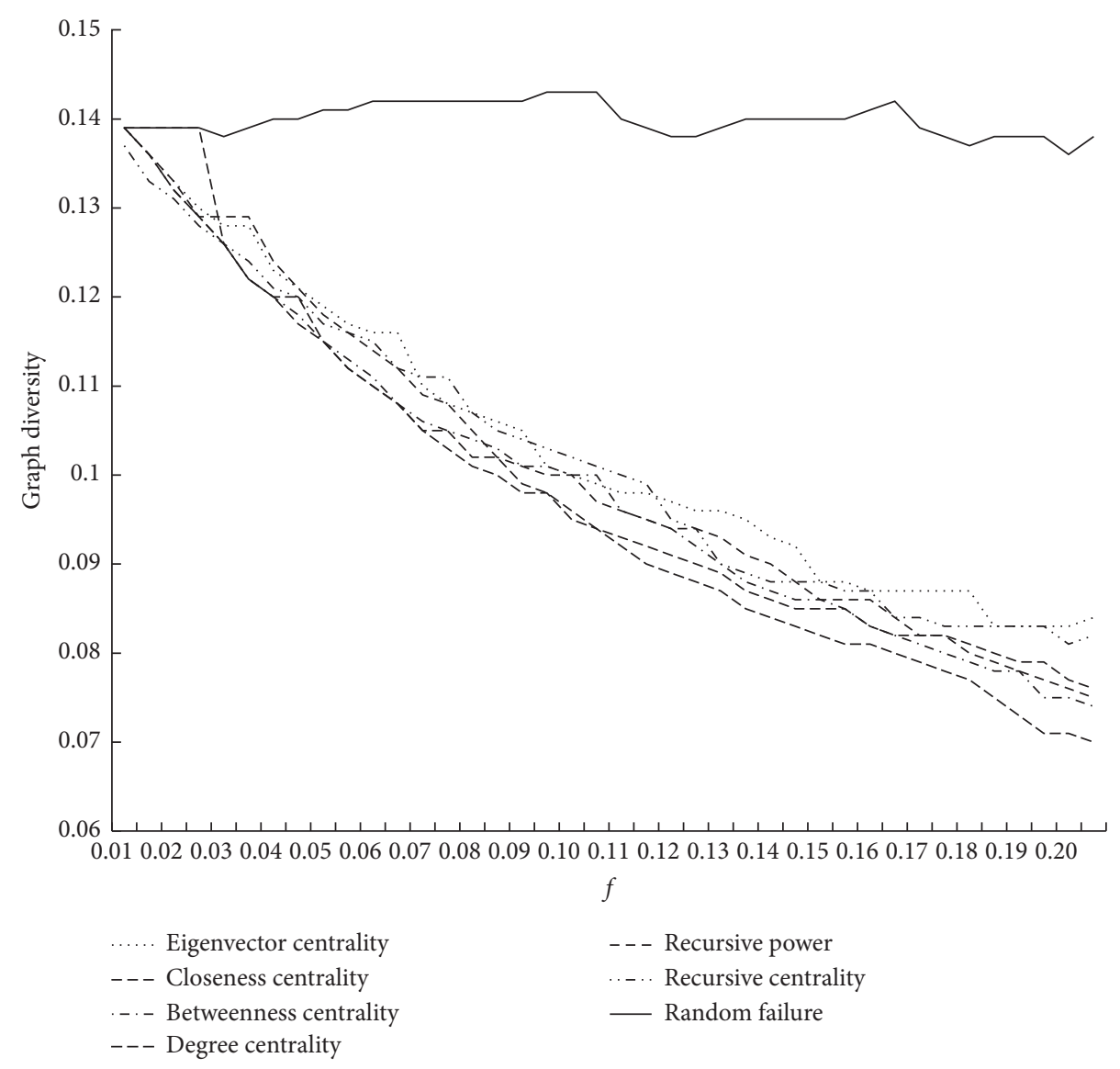

FIGURE 7: Evolution of graph diversity in unweighted and weighted networks.

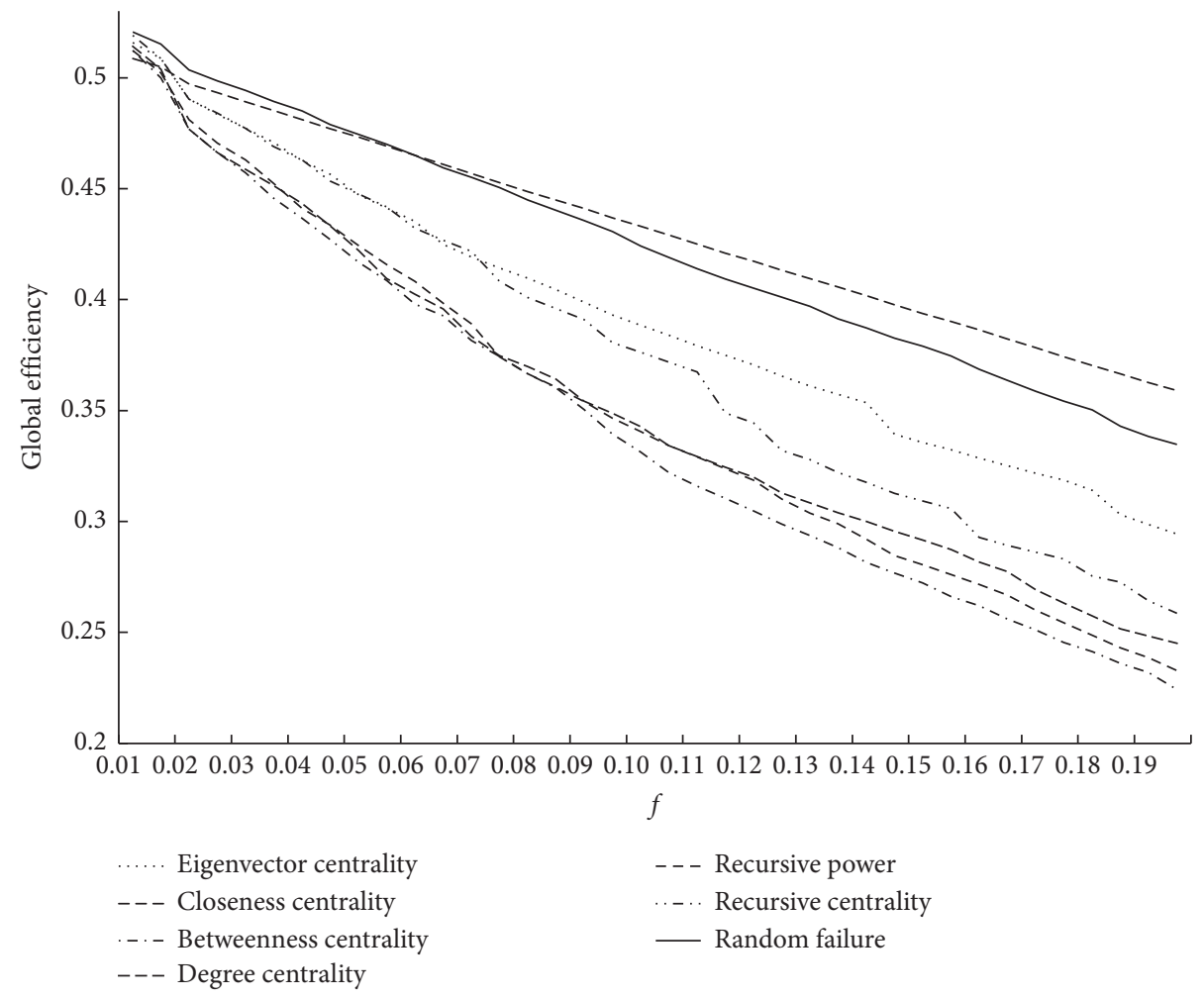

FIGURE 8: Evolution of global efficiency in unweighted and weighted networks. 
TABLE 3: Comparison of robustness metrics in unweighted and weighted approaches.

\begin{tabular}{llcr}
\hline Robustness metric & Unweighted approach & Weighted approach & All \\
\hline Clustering coefficient & Betweenness centrality & Recursive power & Recursive power \\
Average shortest path length & Betweenness centrality & Recursive power & Betweenness centrality \\
Graph diversity & Degree centrality & Recursive power & Degree centrality \\
Global efficiency & Betweenness centrality & Recursive power & Betweenness centrality \\
\hline
\end{tabular}

than those following other rankings. The results were consistent with those of the previous studies where the nodes with high betweenness centrality were critical, especially in terms of shortest path length and global efficiency [43, 44]. As for network robustness in the weighted approach, nodes ranking in recursive power sequence have been identified as critical in the preservation of network functionalities [23]. The results responded well to the correlation analysis that recursive power had the highest correlation relationship with betweenness centrality. This can be explained by tracking its original conception-recursive power captures the extent to which one node dominates its neighbors [45], and betweenness centrality measures the number of short paths between nodes while they pass through a given node [46]. To some extent, both indexes present the transiting property in networks, which determine network robustness from aspects of clustering coefficient, average shortest path length, and global efficiency. Thus, the failure of these high recursive power and betweenness centrality nodes cause their weak neighbors to become disconnected and caused the network functionalities to decrease by a great extent.

This analysis suffers from limitations in terms of the dataset used, which might have shed new light on further scope for future studies. One limitation is the reliability of the flight data pertaining to the ATNBR. Weekly flight data was applied in this study, instead of the more accurate passenger flow. Neither the size of the airplanes nor the origin-destination flow was considered here. Although actual physical movements were reflected by the weekly flights to some extent, passenger flow data would be more valuable for comprehending the social and economic processes involved [47].

\section{Conclusion}

This paper compared the robustness metrics in the application of unweighted and weighted approaches. The main findings of this analysis are as follows. (a) In the network constructed as an unweighted one, betweenness, and degree centrality made more significant contributions in preserving network functionalities as compared to eigenvector and closeness centrality. (b) In the network constructed as a weighted one, recursive power had a higher-level role in preserving network functionalities than recursive centrality. (c) No particular centrality measurement had a significant advantage in representing the totality of robustness. The betweenness centrality sequence was sensitive to the average shortest path length and global efficiency while the recursive power sequence was sensitive to the clustering coefficient, and degree centrality was sensitive to graph diversity.
Studies cited in the current literature related robustness to centrality measurements such as betweenness and closeness and, thus, were limited to the unweighted approach $[48,49]$. While sophisticated properties in the networks were detected, empirical studies called for the uninformed analysis in both weighted and unweighted approaches. In this context, our analysis is a further step toward identifying centrality sequences for determining which one provided the most robustness in unweighted and weighted approaches.

The ultimate aim of exploring aviation systems is to improve the efficiency of travel and optimize the underlying structures [50]. Since air transport can achieve its benefits only when it is available uninterruptedly, increasing the network robustness of ATNBR can improve air transportation security and its ability to effectively respond to emergencies. Thus, the policy implication of this analysis is to construct air transport infrastructure rationally instead of through 'sprawl investment'. Significantly, our analysis found that the nodes-rankings of betweenness centrality, degree centrality, and recursive power sequences reflect different aspects of robustness. This result is important in deciding which nodes should be safeguarded to maintain a given robustness metric against the targeted localized attacks. For instance, nodes with high betweenness centrality such as Moscow, Dubai, Istanbul, among others are critical in preserving global efficiency, while nodes with high degree, such as Dubai, Beijing, and Moscow, are critical for preserving graph diversity. Understanding this allows us to determine which nodes make the network most susceptible to external influences. It also suggests which nodes to protect more stringently from perturbations to preserve the dynamic robustness of the entire network. Thus, we believe our analysis would make useful contributions to the development of appropriate policies.

\section{Data Availability}

The data collected during the study are freely available from Google flight search.

\section{Conflicts of Interest}

The authors declare that they have no conflicts of interest.

\section{Authors' Contributions}

All authors contributed equally to this work. In particular, Lu Zhang developed the original idea for the study and conceived of and designed the methodology. Lu Zhang drafted the manuscript, which was revised by Yannan Zhao, 
Dongli Chen, and Xinhuan Zhang. All authors have read and approved the final manuscript.

\section{Acknowledgments}

This work was supported by the Strategic Priority Research Program of Chinese Academy of Sciences (XDA20040402), China Postdoctoral Science Foundation (2020M680660), and Research Foundation for Youth Scholars of Beijing Technology and Business University (QNJJ2021-56).

\section{References}

[1] J. H. Choi, G. A. Barnett, and B.-S. Chon, "Comparing world city networks: a network analysis of Internet backbone and air transport intercity linkages," Global Networks, vol. 6, no. 1, pp. 81-99, 2006.

[2] J. Chi and J. Baek, "Dynamic relationship between air transport demand and economic growth in the United States: a new look," Transport Policy, vol. 29, pp. 257-260, 2013.

[3] M. Marazzo, R. Scherre, and E. Fernandes, "Air transport demand and economic growth in Brazil: a time series analysis," Transportation Research Part E: Logistics and Transportation Review, vol. 46, no. 2, pp. 261-269, 2010.

[4] Oxford Economic, The Contribution of Air Transport to Sustainable Development in Africa, Air Transport Action Group, Geneva, Switzerland, 2003.

[5] S. Arianos, E. Bompard, A. Carbone, and F. Xue, "Power grid vulnerability: a complex network approach," Chaos: An Interdisciplinary Journal of Nonlinear Science, vol. 19, no. 1, 013119 pages, 2009.

[6] O. Lordan, J. M. Sallan, and P. Simo, "Study of the topology and robustness of airline route networks from the complex network approach: a survey and research agenda," Journal of Transport Geography, vol. 37, pp. 112-120, 2014.

[7] M. Zanin and F. Lillo, "Modelling the air transport with complex networks: a short review," The European Physical Journal Special Topics, vol. 215, no. 1, pp. 5-21, 2013.

[8] M. Janić, "Modelling the resilience, friability and costs of an air transport network affected by a large-scale disruptive event," Transportation Research Part A: Policy and Practice, vol. 71, pp. 1-16, 2015.

[9] A. Bombelli, "Integrators' global networks: a topology analysis with insights into the effect of the COVID-19 pandemic," Journal of Transport Geography, vol. 87, 2020.

[10] Y. Zhou, J. B. Sheu, and J. Wang, "Robustness assessment of urban road network with consideration of multiple hazard events," Risk Analysis, vol. 37, no. 3, 2017.

[11] C. Ducruet and I. Lugo, "Structure and dynamics of transportation networks: models, methods and applications," in The SAGE Handbook of Transport Studies, pp. 347-364, SAGE, New York, NY, USA, 2013.

[12] S. Boccaletti, G. Bianconi, R. Criado et al., "The structure and dynamics of multilayer networks," Physics Reports, vol. 544, no. 1, pp. 1-122, 2014.

[13] A. Bombelli, B. F. Santos, and L. Tavasszy, "Analysis of the air cargo transport network using a complex network theory perspective," Transportation Research Part E: Logistics and Transportation Review, vol. 138, 2020.

[14] R. Albert and A.-L. Barabási, "Topology of evolving networks: local events and universality," Physical Review Letters, vol. 85, no. 24, pp. 5234-5237, 2000.
[15] A. Broder, R. Kumar, F. Maghoul et al., "Graph structure in the web," Computer Networks, vol. 33, no. 1-6, pp. 309-320, 2000.

[16] O. Lordan, J. M. Sallan, P. Simo, and D. Gonzalez-Prieto, "Robustness of the air transport network," Transportation Research Part E: Logistics and Transportation Review, vol. 68, pp. 155-163, 2014.

[17] W. Liu, X. Li, T. Liu, and B. Liu, "Approximating betweenness centrality to identify key nodes in a weighted urban complex transportation network," Journal of Advanced Transportation, vol. 2019, Article ID 9024745, 8 pages, 2019.

[18] S. Lv, Robustness Analysis of Aviation Logistics System Based on Complex Network Theory, Jilin University, Jilin, China, 2014.

[19] D. T. Nguyen, Y. Shen, and M. T. Thai, "Detecting critical nodes in interdependent power networks for vulnerability assessment," IEEE Transactions on Smart Grid, vol. 4, no. 1, pp. 151-159, 2013.

[20] A. Barrat, M. Barthelemy, R. Pastor-Satorras, and A. Vespignani, "The architecture of complex weighted networks," Proceedings of the National Academy of Sciences, vol. 101, no. 11, pp. 3747-3752, 2004.

[21] R. Guimera, S. Mossa, A. Turtschi, and L. A. N. Amaral, "The worldwide air transportation network: anomalous centrality, community structure, and cities' global roles," Proceedings of the National Academy of Sciences, vol. 102, no. 22, pp. 7794-7799, 2005.

[22] Z. He, S. Liu, and M. Zhan, "Dynamical robustness analysis of weighted complex networks," Physica A: Statistical Mechanics and Its Applications, vol. 392, no. 18, pp. 4181-4191, 2013.

[23] L. Zhang, H. Du, X. Zhang, P. De Maeyer, B. Dessein, and Y. Zhao, "Robustness and edge addition strategy of air transport networks: a case study of "the belt and road"” IEEE Access, vol. 7, pp. 96470-96477, 2019.

[24] W. A. Callahan, "China's belt and road initiative and the new eurasian order," Norwegian Institute of International Affairs (NUPI), vol. 22, pp. 1-4, 2016.

[25] T. Summers, "China's 'New silk roads': sub-national regions and networks of global political economy," Third World Quarterly, vol. 37, no. 9, pp. 1628-1643, 2016.

[26] A. D. Cristea, "Buyer-seller relationships in international trade: evidence from U.S. States' exports and business-class travel," Journal of International Economics, vol. 84, no. 2, pp. 207-220, 2011.

[27] P. Dicken, Global Shift: Mapping the Changing Contours of the World Economy, SAGE Publications, New York, NY, USA, 2015.

[28] M. Storper and A. J. Venables, "Buzz: face-to-face contact and the urban economy," Journal of Economic Geography, vol. 4, no. 4, pp. 351-370, 2004.

[29] J. Wang, H. Wang, and J. Jiao, “China's international aviation transport to the belt and road initiative area," Progress in Physical Geography, vol. 34, pp. 554-562, 2015.

[30] L. Zhang, H. Du, Y. Zhao, P. De Maeyer, B. Dessein, and $\mathrm{X}$. Zhang, "Drawing topological properties from a multilayered network: the case of an air transport network in "the belt and road" region," Habitat International, vol. 93, Article ID 102044, 2019.

[31] W. Liu, "Scientific understanding of the belt and road initiative of China and related research themes," Progress in Geography, vol. 34, pp. 538-544, 2015.

[32] G. Pirie, "Trajectories of North-South city Inter-relations," Urban Studies, vol. 47, no. 9, 2010. 
[33] S. Iyer, T. Killingback, B. Sundaram, and Z. Wang, "Attack robustness and centrality of complex networks," PLoS One, vol. 8, no. 4, Article ID e59613, 2013.

[34] P. Bonacich and P. Lloyd, "Eigenvector-like measures of centrality for asymmetric relations," Social Networks, vol. 23, no. 3, pp. 191-201, 2001.

[35] Z. Neal, "Differentiating centrality and power in the world city network," Urban Studies, vol. 48, no. 13, pp. 2733-2748, 2011.

[36] Y. Zhou, J. Wang, and G. Q. Huang, "Efficiency and robustness of weighted air transport networks," Transportation Research Part E: Logistics and Transportation Review, vol. 122, 2019.

[37] D. J. Watts and S. H. Strogatz, "Collective dynamics of 'smallworld' networks," Nature, vol. 393, no. 6684, pp. 440-442, 1998.

[38] Y. Zhou, J. Wang, and H. Yang, "Resilience of transportation systems: concepts and comprehensive review," IEEE Transactions on Intelligent Transportation Systems, vol. 20, no. 12, pp. 4262-4276, 2019.

[39] J. P. Rohrer, A. Jabbar, and J. P. G. Sterbenz, "Path diversification for future internet end-to-end resilience and survivability," Telecommunication Systems, vol. 56, no. 1, 2014.

[40] V. Latora and M. Marchiori, "Efficient behavior of smallworld networks," Physical Review Letters, vol. 87, no. 19, 2001.

[41] P. Angeloudis, K. Bichou, and M. G. H. Bell, "Security and reliability of the liner container-shipping network: analysis of robustness using a complex network framework," in Risk Management in Port Operations, Logistics and Supply Chain Security, pp. 129-140, Informa Law from Routledge, New York, NY, USA, 2013.

[42] D. Bing, "Reliability analysis for aviation airline network based on complex network," Journal of Aerospace Technology and Management, vol. 6, no. 2, pp. 193-201, 2014.

[43] R. Guimerà and L. A. N. Amaral, "Modeling the world-wide airport network," Physics of Condensed Matter, vol. 387540, pp. 381-385, 2004.

[44] L. Gulyas, G. Horvath, T. Cseri, Z. Szakolczy, and G. Kampis, "Betweenness centrality dynamics in networks of changing density," in Proceedings of the 19th International Symposium on Mathematical Theory of Networks and Systems (MTNS 2010), pp. 1091-1098, Budapest, Hungary, July 2010.

[45] Z. Neal, "Does world city network research need eigenvectors?” Urban Studies, vol. 50, no. 8, pp. 1648-1659, 2013.

[46] C. Perez and R. Germon, "Graph creation and analysis for linking actors: application to social data," in Automating open source intelligence: algorithms for OSINTElsevier Inc, Amsterdam, Netherlands, 2016.

[47] Z. Neal, "The devil is in the details: differences in air traffic networks by scale, species, and season," Social Networks, vol. 38, pp. 63-73, 2014.

[48] H.-L. Liu, C. Ma, B.-B. Xiang, M. Tang, and H.-F. Zhang, "Identifying multiple influential spreaders based on generalized closeness centrality," Physica A: Statistical Mechanics and Its Applications, vol. 492, pp. 2237-2248, 2018.

[49] L.-L. Ma, C. Ma, H.-F. Zhang, and B.-H. Wang, "Identifying influential spreaders in complex networks based on gravity formula," Physica A: Statistical Mechanics and Its Applications, vol. 451, pp. 205-212, 2016.

[50] J. Lin and Y. Ban, "The evolving network structure of US airline system during 1990-2010," Physica A: Statistical Mechanics and Its Applications, vol. 410, pp. 302-312, 2014. 